Effect of a working memory training on aspects of cognitive ability and driving ability of older drivers: merits of an adaptive training over a non-adaptive training

Peer-reviewed author version

CUENEN, Ariane; JONGEN, Ellen; BRIJS, Tom; BRIJS, Kris; RUITER, Rob; Houben, Katrien \& WETS, Geert (2016) Effect of a working memory training on aspects of cognitive ability and driving ability of older drivers: merits of an adaptive training over a non-adaptive training. In: TRANSPORTATION RESEARCH PART F-TRAFFIC PSYCHOLOGY AND BEHAVIOUR, 42(1), p. 15-27.

DOI: $10.1016 / j . t r f .2016 .06 .012$

Handle: http://hdl.handle.net/1942/22680 


\title{
Effect of a working memory training on aspects of cognitive ability and driving ability of older drivers: Merits of an adaptive training over a non- adaptive training.
}

\author{
Ariane Cuenen $^{1 *}$, Ellen M.M. Jongen ${ }^{1}$, Tom Brijs ${ }^{1}$, Kris Brijs ${ }^{1,2}$, Katrijn Houben ${ }^{3}$, Geert Wets ${ }^{1}$ \\ ${ }^{1}$ Transportation Research Institute (IMOB), Hasselt University, Diepenbeek, Belgium \\ ${ }^{2}$ Faculty of Applied Engineering Sciences, Hasselt University, Diepenbeek, Belgium \\ ${ }^{3}$ Faculty of Psychology and Neuroscience, Maastricht University, the Netherlands \\ * Corresponding author. \\ E-mail address: ariane.cuenen@uhasselt.be \\ Telephone number: +32(0) 11269166
}

\begin{abstract}
Working memory (WM), important for driving, declines with age. It was investigated whether a WM training would enhance aspects of cognitive ability and driving ability of older drivers. Thirty-eight drivers (mean age 71 years) were randomly assigned to an adaptive WM training $(n=19)$ or a nonadaptive WM training $(n=19)$. In addition, a no-training control group was collected $(n=18)$. During the pre-test and post-test, aspects of cognitive ability and driving ability were assessed. In between, participants in the adaptive training group and the non-adaptive training group conducted a WM training. We hypothesized that improvement on aspects of cognitive ability and driving ability will be largest in the adaptive training group, smaller in the non-adaptive training group and only minimal in the no-training control group. Results indicated that this hypothesis was confirmed for a measure of WM. For two driving measures (i.e., driving speed and reaction to stop signs), group means were in the expected direction, but results were only marginally significant. In addition, there were general test-retest effects for a measure of attention and one driving measure (i.e., gap acceptance). These results are in line with previous cognitive training studies with older people indicating training can improve performance on the trained tasks, but transfer to untrained tasks is only limited. Suggestions for future research are offered.
\end{abstract}

Training; working memory; driving; aging; simulator 


\section{Introduction}

Driving is a complex task that requires cognitive, visual and motor abilities (Eby, Molnar, \& Kartje, 2009). With age, there is a decline of these abilities. With regard to cognitive abilities, increasing age is, among others, characterized by problems of working memory (Eby et al., 2009). Working memory (WM) is the ability to temporarily store or manipulate information (Baddeley, 1992). Previous research of individual differences has indicated the importance of WM for driving in older drivers: Guerrier, Manivannan, and Nair (1999) found that WM was related to decision time while turning left, more specifically, a higher WM score was related to a longer decision time to turn left. In addition, Adrian, Postal, Moessinger, Rascle, and Charles (2011) found that WM was related to a summarized driving measure taking into account speed control, lane position and responses to road signs. More specifically, a higher WM score was related to a better driving performance.

Since driving cessation can lead to social isolation and even depression (Marottoli et al., 1997), there is a need for interventions to keep drivers safe drivers for as long as possible. Cognitive training might serve this purpose. Indeed, several studies have shown that cognitive training targeting older people can improve their cognitive ability (Rebok et al., 2014; Schmiedek, Lövdén, \& Lindenberger, 2010). Hence even people with an advanced age, have considerable plasticity in their cognitive functioning (Kramer \& Willis, 2002). Moreover, a limited number of studies have shown transfer of cognitive training effects to driving ability of older people (Edwards, Delahunt, \& Mahncke, 2009; Roenker, Cissell, Ball, Wadley, \& Edwards, 2003). As a consequence, an increasing number of commercially available cognitive training packages for older people have been introduced that claim to improve driving ability.

Since WM declines with age, it can be expected that a cognitive training specifically targeting WM might improve WM of older people. Indeed, several recent studies showed that WM of older people improved as a consequence of WM training (e.g., Borella, Carretti, Zanoni, Zavagnin, \& De Beni, 2013; Richmond, Morrison, Chein, \& Olson, 2011). Some of these studies showed that WM training also improved other cognitive abilities of older people, like attention (Brehmer, Westerberg, \& Bäckman, 2012; Richmond et al., 2011) and inhibition (Borella et al., 2013). However, there is debate about the (transfer) effects of a WM training (Melby-Lervåg \& Hulme, 2013; Shipstead, Redick, \& Engle, 2012), since several studies found improvement on the trained tasks after following a WM training, but not on untrained tasks (Lange \& Süb, 2015; Zinke, Zeintl, Eschen, Herzog, \& Kliegel, 2012). Interestingly, positive transfer effects of a WM training have been shown in several domains of behavior. For example, after following a WM training, adults showed a decrease of alcoholic drinks intake (Houben, Wiers, \& Jansen, 2011), and children with Attention Deficit Hyperactivity Disorder (ADHD) showed an improvement in behavioral symptoms of ADHD (Klingberg, Forssberg, \& Westerberg, 2002). As for driving, to our knowledge, only two studies have so far investigated whether a computerized WM training improves driving ability of older drivers. First, Cassavaugh and Kramer (2009) investigated the effect of a WM training on specific driving measures like lane position, following distance and accelerator response time to lead-vehicle braking and found a positive effect on response time for the trained group. Second, Seidler, Bernard, Buschkuehl, Jaeggi, Jonides and Humfleet (2010) investigated the effect of a WM training on a summarized driving measure, taking into account speed control, lane position and crashes, under single task and dual task conditions. Although the results were preliminary, because participants were still completing the training, they showed transfer to the summarized driving measure particularly when driving under dual-task conditions. 
The purpose of the present study was to investigate the effect of a computerized WM training on aspects of older drivers' cognitive ability and driving ability. We expected that improvement of cognitive ability and driving ability will be largest in an adaptive training group, smaller in a non-adaptive training group and minimal in a no-training control group. More specifically, the first part of this hypothesis (i.e., improvement in adaptive training group is larger than in non-adaptive training group) is based on previous research that found that an adaptive WM training generally leads to larger training gains than non-adaptive WM training both in older people (Brehmer et al., 2012) and in children (Holmes, Gathercole, \& Dunning, 2009; Karbach, Strobach, \& Schubert, 2015; Klingberg et al., 2002; Klingberg et al., 2005). The final part of this hypothesis (i.e., only minimal improvement in the no-training control group) is based on learning theories that state that participants improve when performing a task for a second time (Boot, Blakely, \& Simons, 2011; Collie, Maruff, Darby, \& McStephen, 2003). Interestingly, a recent WM training study found similar differential group improvement on a WM task for a sample of undergraduates (Lilienthal, Tamez, Shelton, Myerson, \& Hale, 2013).

With regard to cognitive abilities, we expected that the WM training would not only improve WM, but also attention and inhibition. This was based on previous studies that showed an improvement of attention and inhibition after following a WM training (Borella et al., 2013; Brehmer et al., 2012; Richmond et al., 2011) and on the interrelatedness of different cognitive functions and their underlying neural circuits (McNab, Leroux, Strand, Thorell, Bergman, \& Klingberg, 2008). With regard to driving ability, in contrast to investigating the effect of a WM training on a summarized driving measure (Seidler et al., 2010), specific measures of driving ability were used, since previous research has indicated the importance of investigating specific measures (Aksan, Anderson, Dawson, Uc, \& Rizzo, 2015; Anstey \& Wood, 2011; Cuenen et al., 2016; Mullen, Chattha, Weaver, \& Bédard, 2008). Although Cassavaugh and Kramer (2009) already investigated the effect of a WM training on specific driving measures, their selection of driving measures was limited (i.e., lane position, following distance and accelerator response times) and investigated under manipulated circumstances (e.g., during car-following, a visual memory task, a monitoring task, or dual tasking). Therefore, this is the first study to investigate the effect of a WM training on several specific measures of driving ability under normal driving circumstances while encountering situations that are known to be difficult for older drivers (i.e., intersections). Driving measures of interest (i.e., speed control, lane position, gap acceptance while turning left, responses to road signs and crashes) were already investigated as part of a summarized driving measure that was related to WM (Adrian et al. 2011). Here, specific measures and their relations to WM were investigated in an exploratory analysis. Since recent research showed that different driving measures have different underlying cognitive abilities, it can be expected that improvements will be limited to a selection of these driving measures (Aksan et al., 2015; Anstey \& Wood, 2011; Cuenen et al., 2016; Mullen et al., 2008). There were however no a priori expectations which specific driving measures will be affected by the training. The results of this study can guide future initiatives to attenuate declines in cognitive and driving ability in order to extend the independence of older people.

\section{Method}

\subsection{Participants}


Participants aged 60 years or older who were still active drivers, had not had a stroke or sequel in the last six months, had experience with a Personal Computer (PC) ${ }^{1}$ and had a MiniMental State Examination (MMSE) score of 25 or above were recruited. The MMSE is a brief test that examines the cognitive function of an individual. It comprises items assessing orientation to time and place, registration and recall, attention, language and constructional ability (Folstein, Folstein, \& McHugh, 1975). Possible scores range from 0 to 30, with higher scores reflecting higher cognitive ability. Recruitment occurred through the community via (local) media and via oral presentations and flyers distributed in senior associations. Given the possibility of simulator sickness, participants were closely watched for signs of this type of sickness. If a participant showed any signs of simulator sickness, the simulation was immediately terminated and the participant was excluded from further participation.

A sample size of around 25 people per group was chosen based on previous WM training studies that had between 7 and 28 participants per group (Cassavaugh \& Kramer, 2009; Houben et al., 2011; Klingberg et al., 2002). In total, fifty-four participants volunteered. However, sixteen participants dropped out due to simulator sickness $(n=12)$ or personal circumstances (i.e., hospitalization, $n=4)$. After successful completion of the pre-test (i.e., no simulator sickness), participants were randomly assigned to an adaptive WM training $(n=19)$ or a non-adaptive WM training $(n=19)$. In addition, a no-training control group was collected ${ }^{1}$. In total, thirty participants volunteered. However, twelve participants dropped out due to simulator sickness $(n=9)$ or personal circumstances $(n=3)$. Hence, the no-training control group consisted of 18 participants.

The adaptive training group can be regarded as an experimental group. An adaptive training ensures an adequate level of difficulty that does not over- or underchallenge participants. This way, participants continuously work at their individual performance limit (Baltes, Sowarka, \& Kliegl, 1989; Borella et al., 2013; Brehmer et al., 2012; Klingberg et al., 2002; Lange \& Süb, 2015; Richmond et al., 2011). The non-adaptive training group can be regarded as an active control group. In many studies (e.g., Borella et al., 2013; Lange \& Süb, 2015; Richmond et al., 2011; Seidler et al., 2010) this group follows a different training than the experimental group, however, this brings along the disadvantage that it might affect performance differently (Brehmer et al., 2012). In the present study, the adaptive and non-adaptive training groups only differ in terms of task difficulty, as this is kept fixed at a low level in the non-adaptive training group. This provides a conservative assessment of training effects, because the influence of various unspecific factors is attenuated (Brehmer et al., 2012; Shipstead, Redick, \& Engle, 2010; Zehnder, Martin, Altgassen, \& Clare, 2009). The no-training control group can be regarded as a passive control group which allows to determine possible test-retest effects (Brehmer et al., 2012; Chein \& Morrison, 2010; Dahlin, Nyberg, Bäckman, Stigsdotter Neely, 2008; Li, Schmiedek, Huxhold, Röcke, Smith, \& Lindenberger, 2008; Schmiedek et al., 2010).

\subsection{Driving simulator scenario}

Driving ability was measured in a driving simulator since a simulator provides the opportunity to investigate dangerous situations in a standardized, safe and controlled environment (Lee, Cameron, \& Lee, 2003). Furthermore, it allows to investigate specific driving measures, like speed control and lateral position. Recently, positive evidence for simulator validity has been provided (Fisher, Rizzo, Caird, \& Lee, 2011). The study was conducted with STISIM version 2 on a STISIM M400 fixed-base driving simulator with a force-feedback 
steering wheel, an instrumented dashboard, brake and accelerator pedals and with a 135 degree field of view. The visual environment of this simulator is presented on three computer screens (each with $1280 \times 800$ pixels resolution and $60 \mathrm{~Hz}$ refresh rate). Two practice drives preceded the main drive to get acquainted with the driving simulator. In the first practice drive $(2.1 \mathrm{~km})$ almost no curves, no signs, and no other road users were introduced to acquaint drivers with the experience of driving in a simulator. The second practice drive $(5.5 \mathrm{~km})$ was similar to the main drive to acquaint drivers with several traffic situations. The main drive solely consisted of innercity (50 km/hour) segments, and to decrease the risk of simulator sickness did not contain any curves. The main drive included several situations at intersections that are known to be difficult for the older driver, i.e., right of way decisions (Hakamies-Blomqvist, 1993), responses to signs (Bao \& Boyle, 2008; Jongen, Brijs, Brijs, Lutin, Cattersel, \& Wets, 2012) and gap acceptance for turning left (Jongen et al., 2012; Yan, Radwan, \& Guo, 2007). In addition, road hazards occurred during the scenario (i.e., pedestrians suddenly crossing the road without using a pedestrian crossing). The intersections all were a four-way intersection consisting of a straight piece of road and a minor road to the left and to the right.

\subsection{Driving ability}

A total of six specific driving measures were derived for analyses. Although these driving measures are often combined into a summarized measure of driving ability, here we were interested in specific driving measures. These driving measures were selected since previous research found a relation between a summarized version of these measures and WM (Adrian et al., 2011). The first two driving measures, driving speed $(\mathrm{km} / \mathrm{h})$ and standard deviation of lateral lane position (SDLP, m) represent longitudinal and lateral control measures. A measure of longitudinal control (i.e., driving speed) was selected since older drivers compensate for agerelated increases in response time, for example by adopting slower speeds (Fisher et al., 2011). A measure of lateral control (i.e., SDLP) was selected since this measure is an index of roadtracking precision (Ramaekers, 2003), which is considered a reliable characteristic of individual driving performance (O'Hanlon and Ramaekers, 1995; Vuurman, Theunissen, van Oers, van Leeuwen, \& Jolles, 2007; Wester, Bocker, Volkerts, Verster, \& Kenemans, 2008) and provides a sensitive measure of driver impairment (De Waard, 1996; Ramaekers, 2003). Driving speed and SDLP were measured across separate road segments without any events (Trick, Toxopeus, \& Wilson, 2010). The third and fourth driving measure, giving right of way (yes (coded as 1) or no (coded as 0)) and making a complete stop at a stop sign (yes (coded as 1) or no (coded as 0)) were computed from $200 \mathrm{~m}$ before reaching the intersection (with a stop sign) until the location of the intersection (with a stop sign). It was determined whether drivers complied with Belgian traffic regulations that drivers must give right of way at a non-signalized intersection within an inner-city and make a full stop (i.e., speed $=0 \mathrm{~km} / \mathrm{h}$ ) at a stop sign (Bao and Boyle, 2008; Jongen et al., 2012). The fifth driving measure, left turn gap acceptance decision (s), is the time headway between two vehicles on the major road into which a left-turn driver chooses to turn (Jongen et al., 2012; Yan et al., 2007). When the driver approached the intersection, the instruction to turn left was played. On the major road in the opposite lane, a stream of oncoming cars was driving with a speed equaling the speed limit, forcing the driver to make a stop. The first part of the stream consisted of very small gaps (less than $3 \mathrm{~s}$ ) and was followed by the second part of the stream that, similar to Yan et al. (2007), consisted of gaps uniformly increasing in duration from 
$3 \mathrm{~s}$ to $16 \mathrm{~s}$. Participants were asked to indicate their decision to turn left when they judged it was safe to do so by pressing a button. This procedure was followed to minimize the chance of simulator sickness that was very high in a previous study where participants actually made the left turn maneuver (Jongen et al., 2012). These three driving measures (i.e., giving right of way, making a complete stop at a stop sign, and left turn gap acceptance decision) occurred two times at four-way intersections consisting of a straight piece of road and minor roads to the left and to the right. Finally, the number of crashes was assessed. Crashes occurred with road hazards (i.e., pedestrians suddenly crossing the road without using a pedestrian crossing). All driving measures were averaged measures, with the exception of crashes which illustrates the total number of crashes during the drive.

\subsection{Cognitive ability - WM}

WM was measured with the Automated Operation Span (AOSPAN) task (Unsworth, Heitz, Schrock, \& Engle, 2005). This is an adapted version of the original Operation Span (OSPAN) task of Turner and Engle (1989). This task included three practice sessions and one test session. In the first practice session, participants practiced the letter portion of the task. A letter appeared on the screen, and the participants were required to recall the letters in the same order in which they were presented. In the second practice session, participants practiced the math portion of the task. They first saw a math operation, which they needed to solve as quickly as possible. On the next screen a digit was presented and the participants were required to indicate whether it was the correct or false solution of the math operation. After this second practice session, the program calculated each individual's mean time required to solve the math operations. This time (plus 2.5 SD) was then used as a time limit for the math portion of the test session for that individual. In the final practice session, the participants performed both the letter and math portions together, just as they would do in the test session. The participants first saw the math operation and afterwards the letter to be recalled. If the participants took more time to solve the math operations than their average time plus $2.5 \mathrm{SD}$, the program automatically moved on and counted that trial as an error. This served to prevent the participants from rehearsing the letters when they should be solving the operations. After participants completed all practice sessions, they started with the test session, which consisted of three sets of each set size, with set sizes ranging from 3 to 7 . This made for a total of 75 letters and 75 math problems. The order of set sizes was random for each participant. Participants were encouraged to keep their math accuracy at minimum $85 \%$ at all times. The AOSPAN score (i.e., the sum of all perfectly recalled sets) was used as a measure of WM. A higher AOSPAN score corresponds with an improved WM.

\subsection{Cognitive ability - Inhibition}

The Stop Signal Task (SST) was used as a measure of inhibition (Logan \& Cowan, 1984; for a review, see Verbruggen \& Logan, 2008). This task included two practice sessions (each consisting of 40 trials) and one test session (88 trials). In all sessions, participants were required to press a button (left or right) as quickly as possible in response to a stimulus (' $\mathrm{X}$ ' or ' $\mathrm{O}$ ') presented centrally on screen (go trials). In each trial, after $1000 \mathrm{~ms}$, a fixation cross was presented for $500 \mathrm{~ms}$. After this, a stimulus was presented for $1000 \mathrm{~ms}$. The first practice session served to determine the individual's mean Reaction Time (RT), which was used as a reference 
for the second practice session and the test session. The latter sessions consisted of the same task as the first practice session, but in addition, an auditory stimulus $(1000 \mathrm{~Hz}, 70 \mathrm{~dB}, 100 \mathrm{~ms})$ was presented on a randomly selected $25 \%$ of the trials. Upon presentation of this auditory stimulus, the participant needed withheld their response to the stimulus on that trial (stop trials). Importantly, the Stop Signal Delay (SSD; i.e., the time interval between the stimulus and the stop signal) was initially set 50ms below the individual's mean RT. Subsequently the interval varied dynamically, according to a staircase algorithm, to converge on a SSD at which the probability of stopping on stop trials was 50\%. SSD was increased by 50ms if the response was withheld and decreased by 50ms when the response was not withheld. The Stop Signal Reaction Time (SSRT), the time participants need to inhibit their predominant response after hearing the stop signal, was used as a measure of inhibition. This measure can be derived by subtracting the mean SSD from the mean RT. A longer SSRT corresponds with decreased inhibition.

\subsection{Cognitive ability - Attention}

Attention was measured with the Useful Field of View (UFOV). This test was PC-based, with stimuli presented on a 19-inch monitor and responses made using a computer mouse. This version of the UFOV has been shown to be both reliable and valid (Edwards, Vance, Wadley, Cissell, Roenker, \& Ball, 2005). This test consisted of three subtests assessing participants' visual processing speed (UFOV1), divided attention (UFOV2) and selective attention (UFOV3; Ball, Owsley, Sloane, Roenker, \& Bruni, 1993). Scores are expressed in milliseconds, representing the exposure duration required for an observer to perform at an accuracy level of $75 \%$. For each subtest, possible scores range from $16.7 \mathrm{~ms}$ to $500 \mathrm{~ms}$. UFOV-total (i.e., the sum of scores on the three subtests) was used as a measure of attention. Lower scores, correspond with improved attention.

\subsection{WM training}

Participants in both the adaptive training and the non-adaptive training group completed a WM training. The training consisted of three WM subtasks: a visuo-spatial task, a backward digit span task and a letter span task (adapted from Klingberg et al. (2002) and Houben et al. (2011)). During the visuo-spatial task, squares in a 4 x 4 grid changed color one at a time. Participants had to reproduce these squares in the correct order. During the backward digit span task, numbers were presented one at a time, and participants had to reproduce these numbers in reverse order. Finally, in the letter span task, letters were presented one at a time in several circles. One of these circles then changed color, and participants had to enter the corresponding letter. Each task consisted of 30 trials. The training consisted of 25 consecutive sessions spread over at least 25 days. Participants had two days to conduct a session. The session was marked as 'missed' if participants did not complete a session in those two days. Participants could miss maximum 5 sessions. Hence, the total number of sessions varied between 20 and 25 sessions. The training was conducted at home, on a PC, via the internet. Responses were given with a computer mouse or number keys on a keyboard.

In the adaptive training group, the difficulty level of the training was automatically adjusted on a trial-by-trial basis. Initially, each task involved a span of three items. The length of the span changed according to participants' performance. When participants succeed on two consecutive trials, one item was added to the span on the next trial hence task difficulty was 
increased. When participants failed on two consecutive trials, one item was subtracted from the span on the next trial hence task difficulty was decreased. Participants in the adaptive training group could receive a span of maximum 15 items.

In the non-adaptive training group, the difficulty level of the training was not adjusted: each task involved a span of three items. In addition, participants in the non-adaptive training group, started each session with a span of 3 items, whereas participants in the adaptive training group started each session with the number of items of the previous session.

Adherence to the training was monitored for each participant. Each day participants received an automatic invitation mail for a new session. For each day, data was automatically sent to a server if participants finished the session. If no data was received by the server it was marked as "missed". If participants missed a session, they received an automatic mail that they missed the previous session and that they were allowed to only miss five sessions. This overview was checked by the involved researcher. If participants missed two or more sessions, the researcher contacted the person to remind him/her personally of the fact that he/she was allowed to only miss five sessions.

\subsection{Procedure}

First, participants gave informed consent. Then, all participants were screened for cognitive status with the MMSE. After successful completion of the pre-test (i.e., no simulator sickness), participants were randomly assigned to either the adaptive training group $(n=19)$ or the non-adaptive training group $(\mathrm{n}=19)$. In addition, a no-training control group ${ }^{1}$ was collected $(\mathrm{n}=18)$. After the training period, all participants completed a post-test. During the pre- and posttest, participants conducted the cognitive tasks (i.e., AOSPAN, UFOV, and SST) and the driving simulator scenario. In between, participants in the adaptive and non-adaptive training group completed the WM training. After the post-test, participants received a gift certificate. Order of the cognitive tasks was counterbalanced between participants during the pre- and post-test.

\subsection{Data analysis}

The data was processed using SPSS. Before analyses, outliers were treated for each variable. Outliers larger than three standard deviations were replaced with the maximum score within the three standard deviation range (Wood, Anstey, Kerr, Lacherez, \& Lord, 2008).

Univariate analyses of variance (ANOVA) were conducted to check whether there were significant differences between the three groups at pre-test on age, MMSE, the three cognitive measures (i.e., WM, attention and inhibition) and the six driving measures (i.e., driving speed, SDLP, gap acceptance, complete stops at stop signs, giving right of way, and crashes).

A manipulation check was conducted to investigate performance on the training for the two training groups. Performance on the training was assessed by taking the average WM span reached at the end of each training session.

Planned comparisons were conducted to test the hypothesis that improvement of cognitive ability and driving ability will be largest in the adaptive training group, smaller in the non-adaptive training group and minimal in the no-training control group. More specifically, one-way ANOVAs were conducted for each of the dependent measures with the pre-post difference score as the dependent measure (i.e. performance during post-test minus performance during pre-test) and Group (i.e., adaptive training group, non-adaptive training group, no-training 
control group) as the independent measure. We used a linear contrast for each of the dependent measures with the following contrast weights: 10 -1 (Sagi, Tavor, Hofstetter, Tzur-Moryosef, Blumenfeld-Katzir, \& Assaf, 2012).

To investigate whether general test-retest effects were present, repeated measures ANOVAs were conducted for each of the dependent measures to investigate the main effect of Test (pre-test, post-test). In the ANOVA, Test (i.e., pre-test, post-test) served as within-subjects variable and Group (i.e., adaptive training group, non-adaptive training group, no-training control group) served as between-subjects variable. The Greenhouse-Geisser epsilon correction factor was applied to compensate for possible effects of non-sphericity in the measurements compared. Only the corrected F and probability values are reported. An alpha level of .05 was maintained for all statistical tests. A Bonferroni correction was applied to control for repeated comparisons. Effect sizes for the main effect of Test were reported with Cohen's delta. A Cohen's delta of 0.2 indicates a small effect size, 0.5 indicates a medium effect size, and 0.8 indicates a large effect size.

\section{Results}

\subsection{Differences at pre-test}

See Table 1 for the descriptive statistics of the demographic, cognitive and driving measures in the three groups, for the pre- and post-test. At pre-test, participants did not significantly differ in Age, MMSE, on the majority of cognitive measures (i.e., attention, inhibition), or on the majority of driving measures (i.e., driving speed, SDLP, gap acceptance, complete stops at stop signs, and crashes, all $p$-values $>.10$ ). For one cognitive measure (i.e., WM), there was a significant difference between groups $(F(2,51)=6.86, p=.002)$, as participants in the no-training control group had a lower WM capacity than those in the non-adaptive and adaptive training group. For one driving measure (i.e., giving right of way), there was a significant difference between groups $(F(2,53)=8.61, p=.001)$, as participants in the no-training control group gave less right of way than those in the non-adaptive and adaptive training group.

Table 1.

Means and standard deviations (SDs) for the demographic, cognitive and driving measures, in the three groups for the pre-test and post-test.

\begin{tabular}{|l|c|c|c|c|c|c|}
\hline \multicolumn{1}{|c|}{ Measure } & \multicolumn{3}{|c|}{ Pre-test } & \multicolumn{3}{c|}{ Post-test } \\
\hline & $\begin{array}{c}\text { Adaptive } \\
\text { training group }\end{array}$ & $\begin{array}{c}\text { Non-adaptive } \\
\text { training group }\end{array}$ & $\begin{array}{c}\text { No-training } \\
\text { control group }\end{array}$ & $\begin{array}{c}\text { Adaptive } \\
\text { training group }\end{array}$ & $\begin{array}{c}\text { Non-adaptive } \\
\text { training group }\end{array}$ & $\begin{array}{c}\text { No-training } \\
\text { control group }\end{array}$ \\
\hline Demographic measures & Mean (SD) & Mean (SD) & Mean (SD) & Mean (SD) & Mean (SD) & Mean (SD) \\
\hline Age (years) & $70.84(4.66)$ & $69.84(4.39)$ & $73.06(6.20)$ & n.a. & n.a. & n.a. \\
\hline MMSE (number) & $28.74(1.37)$ & $28.74(1.20)$ & $28.72(1.49)$ & n.a. & n.a. \\
\hline Cognitive measures & Mean (SD) & Mean (SD) & Mean (SD) & Mean (SD) & Mean (SD) & Mean (SD) \\
\hline $\begin{array}{l}\text { Working memory } \\
\text { (AOSPAN - number) }\end{array}$ & $18.79(15.17)$ & $20.32(17.44)$ & $3.73(6.87)$ & $29.21(18.18)$ & $27.84(22.81)$ & $4.74(7.50)$ \\
\hline $\begin{array}{l}\text { Attention (UFOV-total - } \\
\text { ms) }\end{array}$ & $240.50(217.63)$ & $204.08(130.57)$ & $332.50(201.85)$ & $187.74(143.47)$ & $130.67(48.60)$ & $293.26(168.86)$ \\
\hline Inhibition (SSRT - ms) & $222.29(46.14)$ & $227.14(50.27)$ & $205.54(50.69)$ & $204.74(50.39)$ & $203.64(50.40)$ & $199.26(54.23)$ \\
\hline
\end{tabular}




\begin{tabular}{|l|c|c|c|c|c|c|}
\hline Driving measures & Mean (SD) & Mean (SD) & Mean (SD) & Mean (SD) & Mean (SD) & Mean (SD) \\
\hline Driving speed $(\mathrm{km} / \mathrm{h})$ & $41.49(4.89)$ & $41.93(6.06)$ & $41.14(6.97)$ & $45.16(5.12)$ & $43.05(5.75)$ & $41.50(6.75)$ \\
\hline SDLP (m) & $0.22(0.05)$ & $0.19(0.05)$ & $0.20(0.06)$ & $0.22(0.07)$ & $0.18(0.03)$ & $0.22(0.06)$ \\
\hline Gap acceptance (s) & $5.36(0.89)$ & $5.54(1.17)$ & $5.68(1.12)$ & $4.93(1.25)$ & $4.96(0.80)$ & $5.18(1.05)$ \\
\hline $\begin{array}{l}\text { Complete stops at stop } \\
\text { signs (0= no/1 = yes) }\end{array}$ & $0.58(0.42)$ & $0.76(0.35)$ & $0.72(0.35)$ & $0.71(0.35)$ & $0.63(0.40)$ & $0.58(0.43)$ \\
\hline $\begin{array}{l}\text { Giving right of way }(0= \\
\text { no/1 = yes) }\end{array}$ & $0.90(0.21)$ & $0.84(0.24)$ & $0.56(0.34)$ & $0.87(0.23)$ & $0.90(0.21)$ & $0.72(0.39)$ \\
\hline Crashes (number) & $0.53(0.70)$ & $0.47(0.70)$ & $0.50(0.62)$ & $0.21(0.42)$ & $0.41(0.72)$ & $0.33(0.59)$ \\
\hline
\end{tabular}

n.a. $=$ not applicable

\subsection{Manipulation check}

On average, 23.05 sessions $(S D=2.32)$ and 23.63 sessions $(S D=1.64)$ out of 25 training sessions were completed by participants in the adaptive training group and the non-adaptive training group, respectively. As shown in Figure 1, performance of participants in the adaptive training group increased on the training sessions during the training period. This is because in this group, the number of items of the training was adjusted adaptively to participants' performance. The error bars show that variability in terms of training improvement is limited. In contrast, in the non-adaptive training group, the number of items of the training was not adjusted adaptively to participants' performance, but fixed to three items.

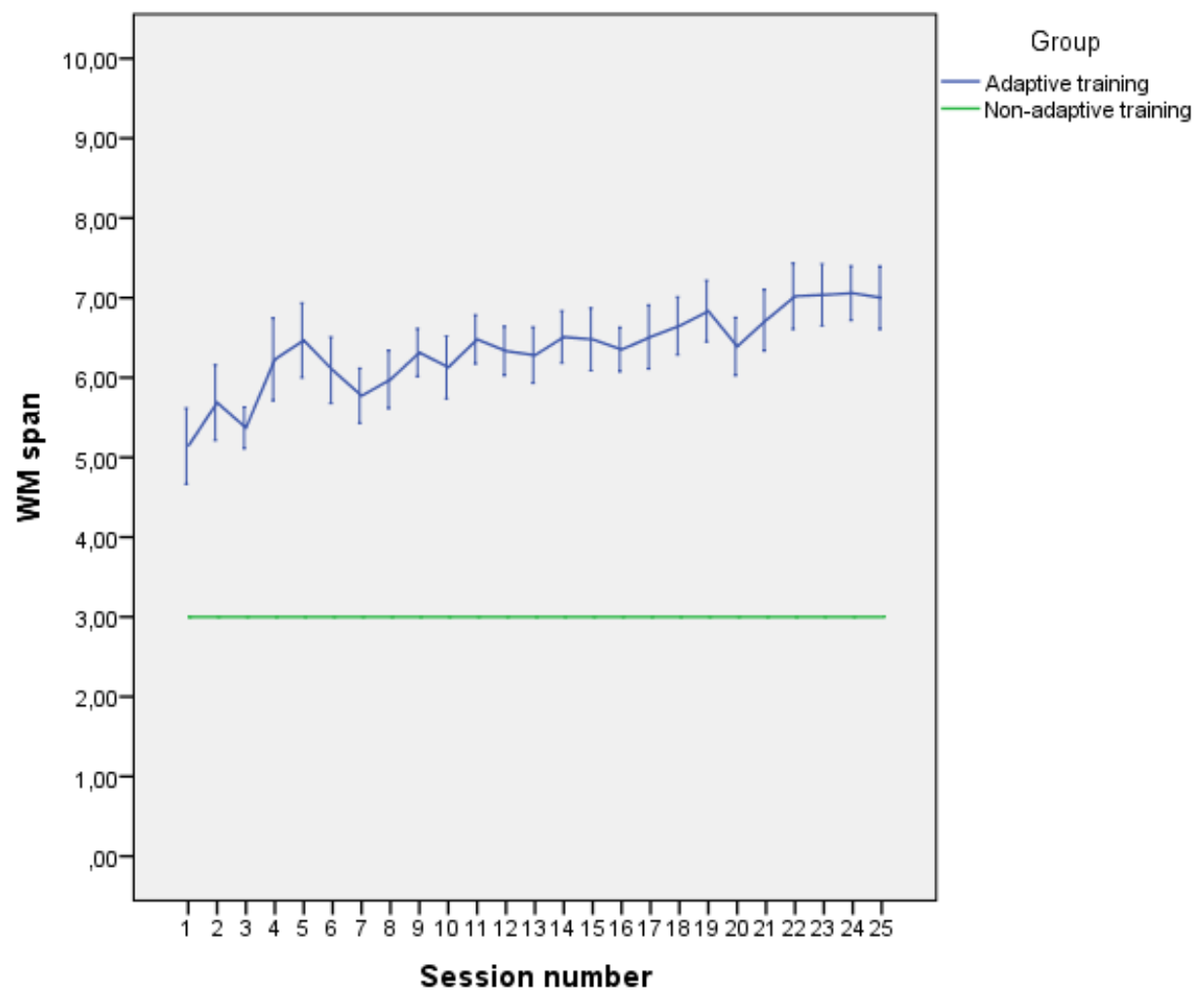

Fig. 1. Mean WM span at the end of each training session of participants in the adaptive training and non-adaptive training groups. WM span was averaged across the three tasks for each training session. Error bars represent standard errors of the mean. 


\subsection{Planned comparisons}

With regard to cognitive ability, as shown in Figure 2, our hypothesis (i.e., improvement will be largest in the adaptive training group, smaller in the non-adaptive training group and only minimal in the no-training control group) was supported for $\mathrm{WM}(\mathrm{t}(21.34)=2.30, \mathrm{p}=.03)$. For the other cognitive measures (i.e., attention and inhibition) there was no support for our hypothesis (all $p$-values >.10).

With regard to driving ability, as shown in Figure 3, effects were in the expected direction, but only marginally significant, for driving speed $(\mathrm{t}(53)=1.96, \mathrm{p}=.06)$ and complete stops at stop signs $(\mathrm{t}(53)=1.87, \mathrm{p}=.07)$. For the other driving measures (i.e., SDLP, gap acceptance, giving right of way, and crashes) there was no support for our hypothesis (all $p$ values >.10).

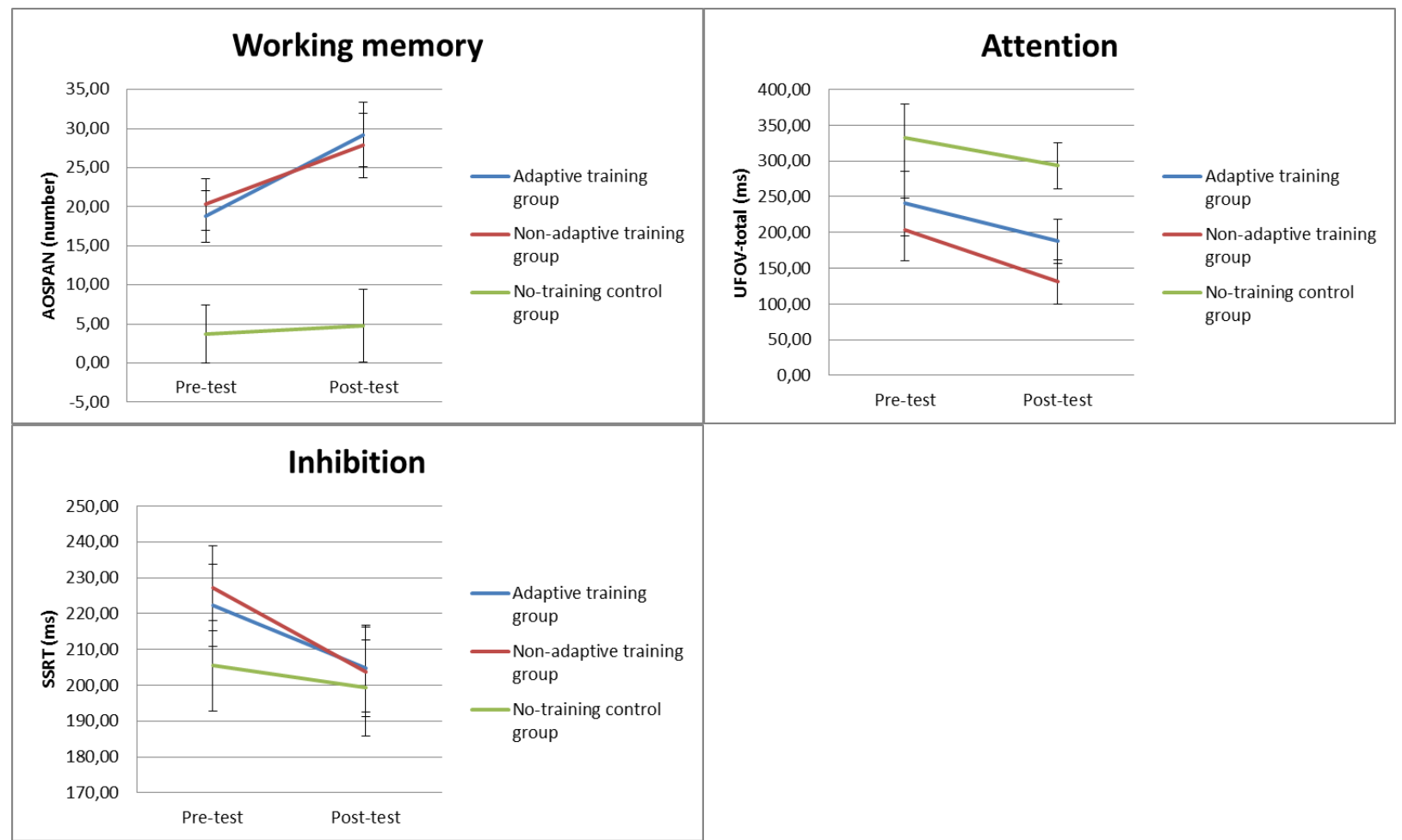

Fig. 2. Estimated marginal means for the cognitive measures at pre-test and post-test, separately for participants in the adaptive training, non-adaptive training and no-training control group. Error bars represent standard errors of the mean. 

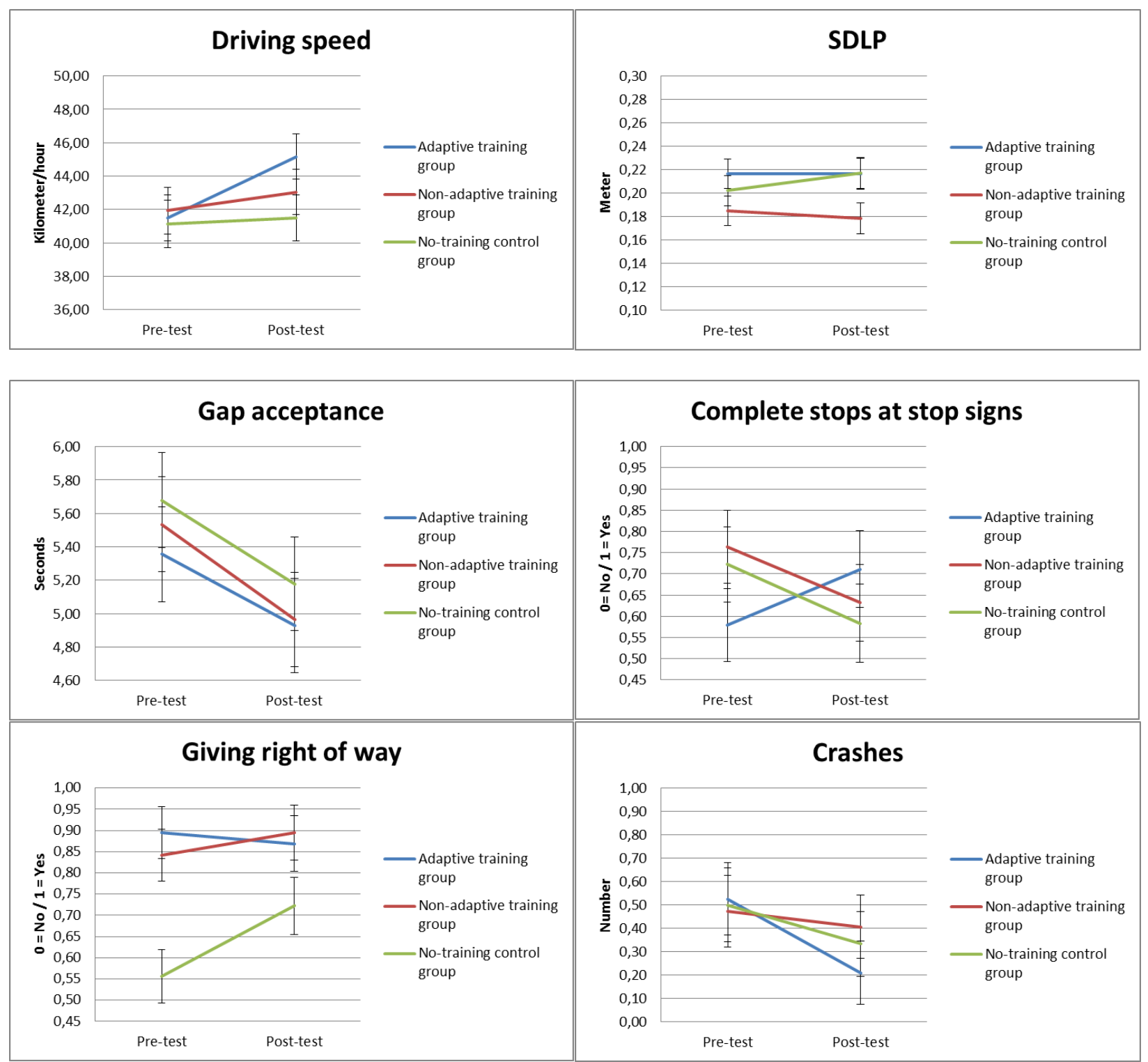

Fig. 3. Estimated marginal means for the driving measures at pre-test and post-test, separately for participants in the adaptive training, non-adaptive training and no-training control group. Error bars represent standard errors of the mean.

\subsection{Repeated measures ANOVA}

See Table 2 for the results of the repeated measures ANOVAs for the cognitive and driving measures. With regard to cognitive ability, there was a significant main effect of Test for the WM and the attention task, indicating a test-retest effect for these cognitive measures. For the inhibition task, there was a marginally significant main effect of Test, indicating that a test-retest effect for this cognitive measure is possible.

With regard to driving ability, there were significant main effects of Test for driving speed and gap acceptance, indicating a test-retest effect for these specific driving measures.

Table 2. 
Corrected $\mathrm{F}$ and probability values per dependent cognitive and driving measure.

\begin{tabular}{|c|c|c|c|}
\hline Measure & $F$ & $p$ & Cohen's d \\
\hline \multicolumn{4}{|l|}{ Cognitive measure } \\
\hline \multicolumn{4}{|c|}{ Working memory (AOSPAN) } \\
\hline Test & 10.41 & $.002 * *$ & 0.37 \\
\hline Test $\mathrm{x}$ condition & 1.90 & .16 & n.a. \\
\hline Condition & 9.87 & $.00 * *$ & n.a. \\
\hline \multicolumn{4}{|l|}{ Inhibition (SSRT) } \\
\hline Test & 3.44 & .07 & n.a. \\
\hline Test $\mathrm{x}$ condition & 0.34 & .72 & n.a. \\
\hline Condition & 0.48 & .62 & n.a. \\
\hline \multicolumn{4}{|l|}{ Attention (UFOV-total) } \\
\hline Test & 10.81 & $.002 * *$ & 0.33 \\
\hline Test $\mathrm{x}$ condition & 0.35 & .70 & n.a. \\
\hline Condition & 4.20 & $.02 * *$ & n.a. \\
\hline \multicolumn{4}{|l|}{ Driving measure } \\
\hline \multicolumn{4}{|l|}{ Driving speed } \\
\hline Test & 6.32 & $.015 * *$ & 0.29 \\
\hline Test $\mathrm{x}$ condition & 2.14 & .13 & n.a. \\
\hline Condition & 0.65 & .53 & n.a. \\
\hline \multicolumn{4}{|l|}{ SDLP } \\
\hline Test & 0.17 & .68 & n.a. \\
\hline Test $\mathrm{x}$ condition & 1.03 & .36 & n.a. \\
\hline Condition & 2.50 & .09 & n.a. \\
\hline \multicolumn{4}{|l|}{ Gap acceptance } \\
\hline Test & 8.47 & $.006 * *$ & 0.48 \\
\hline Test $\mathrm{x}$ condition & 0.06 & .94 & n.a. \\
\hline Condition & 0.36 & .70 & n.a. \\
\hline \multicolumn{4}{|l|}{ Complete stop at stop signs } \\
\hline Test & 0.62 & .43 & n.a. \\
\hline Test $\mathrm{x}$ condition & 2.31 & .11 & n.a. \\
\hline Condition & 0.15 & .86 & n.a. \\
\hline \multicolumn{4}{|l|}{ Giving right of way } \\
\hline Test & 1.71 & .20 & n.a. \\
\hline Test $\mathrm{x}$ condition & 1.28 & .29 & n.a. \\
\hline Condition & 8.06 & $.001 * *$ & n.a. \\
\hline \multicolumn{4}{|l|}{ Crashes } \\
\hline Test & 2.24 & .14 & n.a. \\
\hline
\end{tabular}




\begin{tabular}{|l|l|l|l|}
\hline Test $x$ condition & 0.36 & .70 & n.a. \\
\hline Condition & 0.13 & .88 & n.a. \\
\hline
\end{tabular}

$*<.05, * *<.01$

n.a. = not applicable

\section{Discussion}

The present study investigated whether a WM training would enhance cognitive ability and driving ability of older drivers. Based on previous studies, we hypothesized that improvement of cognitive ability and driving ability would be largest in the adaptive training group, smaller in the non-adaptive training group and only minimal in the no-training control group (Brehmer et al., 2012; Holmes et al. 2009; Karbach et al., 2015; Klingberg et al, 2002; Klingberg et al, 2005; Lilienthal et al., 2013).

More specifically, based on previous studies, we expected (a) that the WM training would improve WM, but also attention and inhibition (Borella et al., 2013; Brehmer et al., 2012; Richmond et al., 2011), and (b) that the WM training would improve several specific measures of driving ability (e.g., speed control, lane position, gap acceptance while turning left, responses to road signs and crashes).

With regard to cognitive ability, after following an adaptive training, older people had a significantly larger improvement of WM, compared to older people following a non-adaptive training or no training. This improvement in WM after following a cognitive training specifically targeting WM is in line with previous research (e.g., Borella et al., 2013; Richmond et al., 2011). It is important to note that although, as indicated by Figure 2, there is a cross-over effect for WM between the adaptive and the non-adaptive training group, the hypothesis of a linear improvement was nevertheless confirmed. Although we found near transfer effects that are a requirement for far transfer effects (Harrison, Shipstead, Hicks, Hambrick, Redick, \& Engle, 2013), we found no differential group improvement for the other cognitive functions. For attention, all groups improved to a similar degree, indicating a general test-retest effect. For inhibition, group means were in the same direction, but results were only marginally significant. A general test-retest effect for this measure is therefore also possible. These results are in line with learning theories that state that participants improve when performing a cognitive task for a second time (Boot et al., 2011; Collie et al., 2003). Moreover, these results are in line with recent studies indicating that cognitive training specifically improves only the cognitive function that was trained (Melby-Lervåg \& Hulme, 2013; Shipstead et al., 2012).

With regard to driving ability, results indicated that for driving speed and reaction to stop signs group means were in the expected direction (i.e. largest improvement with adaptive training, smaller improvement with non-adaptive training and minimal improvement with no training), but results were only marginally significant. It has to be noted that for reactions to stop signs, although the hypothesis of a linear improvement was confirmed, unexpectedly the nonadaptive training group and no-training group deteriorated, showing a lower number of stops in the post-test than in the pre-test. The reason why these groups deteriorated remains unclear. It is known that older drivers typically fail to make a complete stop at stop signs and drive slower (McKnight, 1988). Interestingly, it has been argued that driving slower reflects a way to compensate for the decline in cognitive abilities (Fisher et al., 2011). Our result may be regarded as in line with this, as participants drove faster after improvement of their WM. We found no 
differential group improvement for the other driving measures. Whereas for gap acceptance, all groups improved to a similar degree, indicating a general test-retest effect, for SDLP, giving right of way and crashes, no improvements were found. Taken together, transfer of the improvement of WM to driving performance was only limited. This is in line with recent studies indicating an effect of training solely on the cognitive function being trained, and lack of transfer to daily life activities, like driving (Gaspar, Neider, Simons, McCarley, \& Kramer, 2012; Mayhew, Robertson, \& Vanlaar, 2014). Moreover, improvement of driving after WM improvement was expected for only a selection of driving measures based on recent research showing that different driving measures have different underlying cognitive abilities (Aksan et al., 2015; Anstey \& Wood, 2011; Cuenen et al., 2016; Mullen et al., 2008). Possibly, in order to have an effect on more driving measures, a multifactorial cognitive training is necessary, (i.e., a training that includes tasks addressing a variety of cognitive abilities as used by Schmiedek et al., 2010) or a more direct type of a training targeting driving ability is necessary (e.g. a driving simulator training as used by Casutt, Theill, Martin, Keller, \& Jäncke, 2014).

These results illustrate the importance of investigating training effects on specific measures of driving ability, since summarized measures of driving ability do not provide a detailed view of training effects: whereas the training can have an effect on some aspects of driving, it is possible that it has no effects on other aspects of driving. Indeed, Cassavaugh and Kramer (2009) also only found that improvement was limited to one measure of driving ability (i.e., accelerator response time to lead-vehicle braking) after following a WM training.

Consequently, the same training cannot be used as an intervention for all driving problems. Importantly, effective training programs are tailored to the individual, targeting those specific abilities that are hampered. Hence, our results suggest that those older people that experience difficulty with speed control and responses to stop signs could benefit most from WM training.

\section{Limitations and future research}

Some limitations have to be noted. First, although the investigated training only caused improvements of WM and two driving measures, particularities of the aging population may be responsible for the lack of transfer effects (Lange \& Süb, 2015). Although cognitive plasticity can be assumed even in old age, it is constricted when compared with that of younger people (Lange \& Süb, 2015). Compared to training studies with younger people, training studies with older people have typically reported smaller or non-existent transfer effects (Lange \& Süb, 2015; von Bastian \& Oberauer, 2014). In order to know whether the lack of transfer effects is due to the training or due to the target group, future research should investigate the transfer effects of this WM training in younger people. Since previous studies indicated the relation between WM and driving ability of younger drivers (Mäntylä, Karlsson, \& Marklund, 2009; Ross, Jongen, Brijs, Ruiter, Brijs, \& Wets, 2014), transfer effects of a WM training to aspects of driving ability of younger drivers can be expected. However, since older people are the fastest growing segment of the population and with age there is a decrease in abilities necessary for daily life activities like driving, there is a need for effective interventions for older people to ensure their quality of life. Future initiatives should be explored to attenuate declines in cognitive ability and driving ability. As for cognitive training, in a recent meta-analysis a group-based training consisting of maximum three sessions was advised (Lampit, Hallock, \& Valenzuela, 2015). 
Second, the present study only investigated immediate training effects, therefore the longterm effects remain unclear. Future research should examine the durability of effects. Previous research found maintained effects for several months (Borella et al., 2013; Li et al., 2008).

Third, the training was conducted at home. Although this has several advantages, it reduces experimenter control (i.e., standard testing environment and procedures). Related to this limitation, although the no-training control group received no training, we cannot be sure that this group did not train their WM during the study. However, based on our results that WM improvement was minimal in the no-training control group, there were no indications that people trained their WM.

Fourth, we used a non-adaptive training group, that can be regarded as an active control group, who performs the same training as the adaptive training group but on a fixed low level. This was done to provide a conservative assessment of training effects, because the influence of various unspecific factors is attenuated (Brehmer et al., 2012; Shipstead et al., 2010; Zehnder et al., 2009). Although there are no indications that the non-adaptive training is less stimulating than the adaptive training, since the number of completed sessions is comparable (i.e., 23.05 sessions completed by participants in the adaptive training group and 23.63 sessions completed by participants in the non-adaptive training group), it might be interesting for future research to incorporate an active control group performing an equal amount of training time as the adaptive training group but on tasks that are not expected to improve cognitive or driving ability. This might help to further establish that the specific training under investigation, rather than any challenging training task, is directly related to improvements in cognitive and driving ability (Cassavaugh \& Kramer, 2009).

Fifth, driving ability in the present study was investigated with a driving simulator. Although simulators have several advantages and positive evidence for simulator validity has been provided (Fisher et al., 2011), the present study should be replicated during real-world driving to bolster our conclusions. By replicating the study during real-world driving, the practical relevance of the results can be investigated. Looking at the results, it seems that the adaptive training group (the group who improved the most compared to the other groups) drove $3.67 \mathrm{~km} / \mathrm{h}$ faster and stopped 0.13 times more at a stop sign in the driving simulator after following the training. However, it has to be noted that for the driving simulator, there is evidence for relative validity, not for absolute validity. This means that the direction of change of a simulated driving measure is in the same direction as a corresponding driving measure in the real world, but does not produce the same numerical change. As a consequence, investigating the effect of the training on driving in real-world conditions would cause an increase in speed and number of complete stops, however, the amount of increase can be different than observed in the simulator (Fisher et al., 2011).

Finally, the present study investigated the effect of a WM training on specific measures of driving ability under normal driving circumstances (e.g., not during car-following) while encountering situations that are known to be difficult for older drivers (i.e., intersections). In order to guide future initiatives to increase driving safety of older people, future research should investigate other measures related to driving ability, e.g., gaze behavior. In addition, it would be interesting to further investigate the reasons behind certain driving behaviors. In the present study, participants did not always follow the traffic rules (e.g., not making a complete stop at a stop sign). This could be due to an error or due to a violation of the traffic rule. 


\section{Acknowledgements}

This study was funded by the Policy Research Center for Traffic Safety. The authors want to thank Michiel Vestjens for his technical assistance with the training.

\section{References}

Adrian, J., Postal, V., Moessinger, M., Rascle, N., \& Charles, A. (2011). Personality trait and executive functions related to on-road driving performance among older drivers. Accident Analysis and Prevention, 43, 1652-1659.

Aksan, N., Anderson, S.W., Dawson, J., Uc, E., \& Rizzo, M. (2015). Cognitive functioning differentially predicts different dimensions of older drivers' on-road safety. Accident Analysis and Prevention, 75, 236-244.

Anstey, K.J., \& Wood, J. (2011). Chronological age and age-related cognitive deficits are associated with an increase in multiple types of driving errors in late life. Neuropsychology, 25(5), 613-621.

Baddeley, A. (1992). Working memory. Science, 255, 556-559.

Ball, K.K., Owsley, C., Sloane, M., Roenker, D.L., \& Bruni, J. (1993). Visual attention problems as predictor of vehicle crashes in older drivers. Investigative Ophthalmology and Visual Science, 34, 3110-3123.

Baltes, P.B., Sowarka, D., \& Kliegl, R. (1989). Cognitive training research on fluid intelligence in old age: what can older adults achieve by themselves? Psychol. Aging, 4, 217-221.

Bao, S. \& Boyle, L. (2008). Driver Performance at Two-Way Stop-Controlled Intersections on Divided Highways. Transportation Research Record, 2069, 26-32.

Boot, W.R., Blakely, D.P., \& Simons, D.J. (2011). Do action video games improve perception and cognition? Frontiers in psychology, 226 (2), 1-6.

Borella, E., Carretti, B., Zanoni, G., Zavagnin, M., \& De Beni, R. (2013). Working memory training in old age: an examination of transfer and maintenance effects. Archives of clinical neuropsychology, 28(4), 331-347.

Brehmer, Y., Westerberg, H., \& Bäckman, L. (2012). Working-memory training in younger and older adults: training gains, transfer and maintenance. Frontiers in human neuroscience, 6 (63), 1-7.

Cassavaugh, N. D., \& Kramer, A. F. (2009). Transfer of computer-based training to simulated driving in older adults. Applied Ergonomics, 40, 943-952.

Casutt, G., Theill, N., Martin, M., Keller, M., \& Jäncke, L. (2014). The drive-wise project: driving simulator training increases real driving performance in healthy older drivers. Frontiers in aging neuroscience, 6 (85), 1-14.

Chein, J.M., \& Morrison, A.B. (2010). Expanding the mind's workspace: Training and transfer effects with a complex working memory span task. Psychonomic Bulletin \& Review, 17 (2), 193-199.

Collie, A., Maruff, P., Darby, D. G., \& McStephen, M. (2003). The effects of practice on the cognitive test performance of neurologically normal individuals assessed at brief test-retest intervals. Journal of the International Neuropsychological Society, 9 (3), 419-428.

Cuenen, A., Jongen, E.M.M., Brijs, T., Brijs, K., Lutin, M., Van Vlierden, K., \& Wets, G. (2016). The relations between specific measures of simulated driving ability and functional 
ability: New insights for assessment and training programs of older drivers. Transportation Research Part F, 39, 65-78.

Dahlin, E., Nyberg, L., Bäckman, L., \& Stigsdotter Neely, A., (2008). Plasticity of Executive Functioning in Young and Older Adults: Immediate Training Gains, Transfer, and LongTerm Maintenance. Psychology and Aging, 23 (4), 720-730.

De Waard, D. (1996). The measurement of drivers' mental workload (Unpublished doctoral dissertation). Haren, The Netherlands: University of Groningen Traffic Research Center.

Eby, D., Molnar, L.J., \& Kartje, P.S. (2009). Maintaining safe mobility in an aging society. NW: CRC Press.

Edwards, J.D., Vance, D.E., Wadley, V.G., Cissell, G.M., Roenker, D.L., \& Ball, K. (2005). Reliability and validity of Useful Field of View Test scores as administered by personal computer. Journal of Clinical and Adaptive training Neuropsychology, 27, 529-543.

Edwards, J.D., Delahunt, P.B., \& Mahncke, H.W. (2009). Cognitive speed of processing training delays driving cessation. Journal of Gerontology: Medical sciences, 64 (12), 1262-1267.

Fisher, D.L., Rizzo, M., Caird, J.K., \& Lee, J.D. (2011). Handbook of Driving Simulation for Engineering, Medicine, and Psychology. Eds. Boca Raton, FL: CRC Press/Taylor and Francis.

Folstein, M.F., Folstein S.E., \& McHugh P.R. (1975). Mini-Mental State: a practical method for grading the cognitive state of patients for the clinician. J Psychiatr Res, 12, 189-198.

Gaspar, J.G., Neider, M.B., Simons, D.J., McCarley, J.S., \& Kramer, A.F. (2012). Examining the efficacy of training interventions in improving older driver performance. Proceedings of the human factors and ergonomics society 56th annual meeting, 144-148.

Guerrier, J.H., Manivannan, P., \& Nair, S.N. (1999). The role of working memory, field dependence, visual search, and reaction time in the left turn performance of older female drivers. Applied ergonomics, 30, 109-119.

Hakamies-Blomqvist, L.E. (1993). Fatal accidents of older drivers. Accident Analysis and Prevention, 25, 19-27.

Harrison, T.L., Shipstead, Z., Hicks, K.L., Hambrick, D.Z., Redick, T.S., \& Engle, R.W. (2013). Working Memory Training May Increase Working Memory Capacity but Not Fluid Intelligence. Psychological Science, 1-11.

Holmes, J., Gathercole, S.E., \& Dunning, D.L. (2009). Adaptive training leads to sustained enhancement of poor working memory in children. Developmental Science, 12 (4), F9-F15.

Houben, K., Wiers, R.W., \& Jansen, A. (2011). Getting a grip on drinking behavior: training working memory to reduce alcohol abuse. Psychological science, 22 (7), 968-975.

Jongen, E.M.M., Brijs, T., Brijs, K., Lutin, M., Cattersel, M., \& Wets, G. (2012). The relation between visual attention and specific measures of simulated driving in older drivers. Transportation Research Board Conference Proceedings. Washington, D.C..

Karbach, J., Strobach, T., \& Schubert, T. (2015). Adaptive working-memory training benefits reading, but not mathematics in middle childhood. Child Neuropsychology: A Journal on Normal and Abnormal Development in Childhood and Adolescence, 21 (3), 285-301.

Klingberg, T., Fernell, E., Olesen, P.J., Johnson, M., Gustafsson, P., Dahlström, K., Gillberg, C.G., Forssberg, H., \& Westerberg, H. (2005). Computerized Training of Working Memory in Children With ADHD-A Randomized, Controlled Trial. Journal of American Academy of Child and Adolescent Psychiatry, 44 (2), 177-186.

Klingberg, T., Forssberg, H., \& Westerberg, H. (2002). Training of working memory in children with ADHD. Journal of Clinical and Adaptive training Neuropsychology, 24 (6), 781-791. 
Kramer, A.F., \& Willis, S.L. (2002). Enhancing the cognitive vitality of older adults. Current directions in psychological science, 11 (5), 173-177.

Lampit, A., Hallock, H., \& Valenzuela, M. (2014). Computerized Cognitive Training in Cognitively Healthy Older Adults: A Systematic Review and Meta-Analysis of Effect Modifiers. Plos medicine, 11 (11), 1-18.

Lange, S., \& Süb, H. (2015). Experimental Evaluation of Near- and Far-Transfer Effects of an Adaptive Multicomponent Working Memory Training. Applied Cognitive Psychology, 29, 502-514

Lee, H.C., Cameron, D., \& Lee, A.H. (2003). Assessing the driving performance of older adult drivers: on-road versus simulated driving. Accident analysis and prevention, 35, 797-803.

Li, S., Schmiedek, F., Huxhold, O., Röcke, C., Smith, J., \& Lindenberger, U. (2008). Working memory plasticity in old age: practice gain, transfer and maintenance. Psychology and aging, 23 (4), 731-742.

Lilienthal, L., Tamez, E., Shelton, J.T., Myerson, J., \& Hale, S. (2013). Dual n-back training increases the capacity of the focus of attention. Psychon Bull Rev, 20, 135-141.

Logan, G. D. \& Cowan, W. B. (1984). On the ability to inhibit thought and action: a theory of an act of control. Psychological Review, 91, 295-327.

Mäntylä, T., Karlsson, M.J., \& Marklund, M. (2009). Executive control functions in simulated driving. Applied Neuropsychology, 16, 11-18.

Marottoli, R. A., de Leon, C. F. M., Glass, T. A., Williams, C. S., Cooney, L.M., Berkman, L. F., \& Tinetti, M. E. (1997). Driving cessation and increased depressive symptoms: Prospective evidence from the New Haven EPESE. Journal of the American Geriatrics Society, 45, $202-$ 206.

Mayhew, D., Robertson, R., \& Vanlaar, W. (2014). Computer-based cognitive training programs for older drivers: what research tells us. Traffic Injury Research Foundation. Ottawa, Canada.

McKnight, A.J. (1988). Driver and pedestrian training. Transportation in an aging society: Improving mobility and safety for older persons, Volume 2. Special report 218, 101-133.

McNab, F., Leroux, G., Strand, F., Thorell, L., Bergman, S., \& Klingberg, T. (2008). Common and unique components of inhibition and working memory: An fMRI, within-subjects investigation. Neuropsychologia, 46, 2668-2682.

Melby-Lervåg, M., \& Hulme, C. (2013). Is Working Memory Training Effective? A MetaAnalytic Review. Developmental Psychology, 49 (2), 270-291.

Mullen, N.W., Chattha, H.K., Weaver, B., \& Bédard, M. (2008). Older driver performance on a simulator: associations between simulated tasks and cognition. Advances in Transportation Studies Special Issue, 31-42.

O’Hanlon, J. F., \& Ramaekers, J. G. (1995). Antihistamine effects on actual driving performance in a standard test: A summary of Dutch experience, 1989-94. Allergy, 50, 234-242.

Ramaekers, J. G. (2003). Antidepressants and driver impairment: Empirical evidence from a standard on-the-road test. Journal of Clinical Psychiatry, 64, 20-29.

Rebok, G.W., Ball, K., Guey, L.T., Jones, R.N., Kim, H., King, J.W., Marsiske, M., Morris, J.N., Tennstedt, S.L., Unverzagt, F.W., \& Willis, S.L. (2014). Ten-year effects of the advanced cognitive training for independent and vital elderly cognitive training trial on cognition and everyday functioning in older adults. Journal of the American Geriatrics Society, 62 (1), 1624. 
Richmond, L. L., Morrison, A. B., Chein, J. M., \& Olson, I. R. (2011). Working Memory Training and Transfer in Older Adults. Psychology and Aging, 26, 813-822.

Roenker, D.L., Cissell, G.M., Ball, K., Wadley, V.G., \& Edwards, J.D. (2003). Speed-of processing and driving simulator training result in improved driving performance. Human Factors, 45 (2), 218-233.

Ross, V., Jongen, E., Brijs, T., Ruiter, R., Brijs, K., \& Wets, G. (2014). The Relation Between Cognitive Control and Risky Driving in Young Novice Drivers. Applied Neuropsychology: Adult, 0, 1-12

Sagi, Y., Tavor, I., Hofstetter, S., Tzur-Moryosef, S., Blumenfeld-Katzir, T., \& Assaf, Y. (2012). Learning in the Fast Lane: New Insights into Neuroplasticity. Neuron, 73, 1195-1203.

Schmiedek, F., Lövdén, M., \& Lindenberger, U. (2010). Hundred days of cognitive training enhance broad cognitive abilities in adulthood: findings from the COGITO study. Frontiers in aging neuroscience, 2 (27), 1-10.

Seidler, R. D., Bernard, J. A., Buschkuehl, M., Jaeggi, S., Jonides, J., \& Humfleet, J. (2010). Cognitive training as an intervention to improve driving ability in the older adult (No. MCASTL 2010-01).

Shipstead, Z., Redick, T.S., \& Engle, R. W. (2010). Does working memory training generalize? Psychol. Belg. 50, 245-276.

Shipstead, Z., Redick, T.S., \& Engle, R.W. (2012). Is Working Memory Training Effective? Psychological Bulletin, 138 (4), 628-654.

Trick, L. M., Toxopeus, R., \& Wilson, D. (2010). The effects of visibility, traffic density and navigational challenge on speed compensation and driving performance in older adults. Accident Analysis and Prevention, 42, 1661-1676.

Turner, M. L., \& Engle, R. W. (1989). Is working memory capacity task dependent? Journal of Memory and Language, 28, 127-154.

Unsworth, N., Heitz, R.P., Schrock, J.C., \& Engle, R. (2005). An automated version of the operation span task. Behavior Research Methods, 37 (3), 498-505.

Verbruggen, F., \& Logan, G.D. (2008). Automatic and Controlled Response Inhibition: Associative Learning in the Go/No-Go and Stop-Signal Paradigms. J Exp Psychol Gen., 137 (4), 649-672.

Von Bastian, C.C., \& Oberauer, K. (2014). Effects and mechanisms of working memory training: a review. Psychological Research, 78, 803-820

Vuurman, E., Theunissen, E., van Oers, A., van Leeuwen, C., \& Jolles, J. (2007). Lack of effects between rupatadine $10 \mathrm{mg}$ and placebo on actual driving performance of healthy volunteers. Human Psychopharmacology: Clinical and Experimental, 22, 289-297.

Wester, A. E., Bocker, K. B., Volkerts, E. R., Verster, J. C., \& Kenemans, J. L. (2008). Eventrelated potentials and secondary task performance during simulated driving. Accident Analysis and Prevention, 40, 1-7.

Wood, J. M., Anstey, K. J., Kerr, G. K., Lacherez, P. F., \& Lord, S. (2008). A multidomain approach for predicting older driver safety under in-traffic road groups. Journal of the American Geriatrics Society, 56 (6), 986-993.

Yan, X., Radwan, E., \& Guo, D. (2007). Effects of major-road vehicle speed and driver age and gender on left-turn gap acceptance. Accident Analysis and Prevention, 39, 843-852.

Zehnder, F., Martin, M., Altgassen, M., \& Clare, L. (2009). Memory training effects in old age as markers of plasticity: a meta-analysis. Restor. Neurol.Neurosci., 27, 507-520. 
Zinke, K., Zeintl, M., Eschen, A., Herzog, C., \& Kliegel, M. (2012). Potentials and Limits of Plasticity Induced by Working Memory Training in Old-Old Age. Gerontology, 58, 79-87.

\footnotetext{
${ }^{1}$ Although procedure and time between testing was the same for all three groups, participants of the no-training control group were later recruited compared to participants of the two training groups (i.e., adaptive and nonadaptive) and 'experience with a PC' was not added as an inclusion criteria. Unexpectedly, average OSpan scores of the no-training control group were lower when compared to those of the adaptive training group and the nonadaptive training group. Closer inspection of the data showed that this is due to a higher frequency of zero-scores in the no-training control group $(n=10)$, than in the adaptive training group $(n=2)$ or the non-adaptive training group ( $n=4)$. Possibly this is due to later recruitment of the no-training control group. As a consequence, participants were randomized across the two training groups (i.e., adaptive and non-adaptive), thereby distributing zero-scores across the two groups, whereas all low scorers were assigned to the same no-training control group. In addition, this can be due to the difference in recruitment, since for the no-training control group, experience with a PC was not added as an inclusion criterion. There were no significant differences between scores in the notraining control group on each of the cognitive tests for the pre-test and post-test, which supports that the lower OSpan scores are not due to measurement errors.
} 